\title{
Escala de Calidad de Vida (ECV) para personas con discapacidad que envejecen: estudio preliminar ${ }^{1}$
}

\section{The Quality of Life Questionnaire (QLQ) for persons with disability who are ageing: preliminary study}

\author{
M. a ÁNGELES ALCEDO RODRÍGUEZ* \\ ANTONIO-LEÓN AGUADO DÍAZ* \\ BENITO ARIAS MARTÍNEZ** \\ MENI GONZÁLEZ GONZÁLEZ* \\ CRISTINA ROZADA RODRÍGUEZ*
}

\begin{abstract}
RESUMEN
El objetivo de este trabajo es la elaboración de un instrumento para evaluar la calidad de vida (CV) de las personas con discapacidad que envejecen. Se ha aplicado a una muestra de 2.292 personas con discapacidad mayores de 45 años procedentes de distintas comunidades autónomas del territorio español. La necesidad de disponer de instrumentos de evaluación de CV para este colectivo de personas con discapacidad que envejecen, que nos permitan realizar medidas fiables y válidas sobre las que planificar actuaciones $y$ estrategias de intervención eficaces, ha orientado la elaboración de esta Escala de Calidad de Vida (ECV). El análisis de las propiedades psicométricas de la escala muestra una elevada consistencia interna, alfa de Cronbach de 0,92. La dimensionalidad de la escala arroja una solución hexafactorial que muestrea seis de las principales dimensiones de CV, en concreto, bienestar fisico, inclusión social, bienestar emocional, desarrollo personal y autodeterminación, relaciones interpersonales y bienestar material. Estos resultados evidencian la adecuación de la ECV para evaluar la CV tal y como es percibida por la muestra de nuestro estudio, las personas con discapacidad que envejecen.
\end{abstract}

\footnotetext{
* Universidad de Oviedo.

** Universidad de Valladolid.
} 


\title{
PALABRAS CLAVE
}

Envejecimiento, Discapacidad, Evaluación, Calidad de vida.

\begin{abstract}
The goal of this paper is the elaboration of an instrument to assess quality of life (QL) of ageing persons with disability. It has been applied to a sample of 2.292 persons older than 45 years, from different regions of Spain. The need of $Q L$ assessment instruments for this group of persons with disability who are ageing will allow us to make valid and reliable measures to plan efficient intervention actions and strategies, and has motivated the creation of this Quality of Life Questionnaire (QLQ). The analysis of the scale psychometric properties shows high internal consistency, Cronbach's alfa of 0,92. Scale dimensionality offers a hexafactorial solution that tests six of the main QL dimensions, such as, physical well being, social inclusion, emotional well being, personal development and self-determination, interpersonal relationship and material well being. These results give evidence of the QLL suitability to assess the QL, as it is perceived by the sample of our study, the ageing persons with disability.
\end{abstract}

\section{KEY WORDS}

Ageing, Disability, Evaluation, Quality of life. 


\section{INTRODUCCIÓN}

El concepto de calidad de vida (CV), que en la época moderna nace en referencia a la eficacia de los tratamientos con la pretensión de llegar a estimaciones acerca de las pérdidas sufridas como consecuencia de la enfermedad, no es un concepto nuevo, ya se encuentra implícito en el pensamiento presocrático, aunque no surge de forma explícita hasta hace unos 30 años, aproximadamente. El carácter dinámico de este concepto y su conexión con diversos objetivos hacen que su definición constituya un difícil reto. No existe una definición unánimemente aceptada de CV, sino multitud de ellas con diferentes puntos en común. Ya hace años se hablaba de la existencia de más de 100 formulaciones y medidas del constructo (Keith, 2001). Esta pluralidad conceptual también está presente en el campo de la discapacidad. Así, las distintas definiciones de este término tienen en común el recoger un amplio espectro de dimensiones de la experiencia humana, dimensiones que tienen que ver con aspectos variados, relativos a la relación del individuo con su vida (en qué medida las expectativas se aproximan a los logros conseguidos), a factores objetivos y subjetivos que impactan sobre la vida o ámbitos de la experiencia humana, y al grado de satisfacción con las circunstancias percibidas de la vida (Aguado, González, Alcedo y Arias, 2003).

El modelo de CV para el ámbito de la discapacidad más aceptado por la comunidad científica internacional es el propuesto por Schalock (1996), revisado en fechas recientes (Schalock y Verdugo, 2006). Dicho concepto utiliza indicadores referidos a ocho dimensiones que representan el núcleo de las necesidades fundamentales de la vida de cada uno: bienestar emocional, relaciones interpersonales, bienestar material, desarrollo personal, bienestar fisico, autodeterminación, inclusión social y derechos. La preocupación por el bienestar físico, especialmente por la salud y la asistencia sanitaria, resultan los indicadores más destacados y determinantes de la CV en las personas con discapacidad que envejecen. Estos indicadores aparecen recogidos en más del $80 \%$ de los estudios centrados en el proceso de envejecimiento de las personas con discapacidad. La explicación viene dada por el hecho de que la percepción del sujeto se modifica radicalmente cuando presenta graves problemas de salud. Junto a estos indicadores, el lugar de residencia y sus características, por un lado, y los recursos de apoyo social existentes, el papel de la familia y de los proveedores de servicios, por otro, aparecen como componentes vitales de la CV. La inclusión social, es otro de los indicadores también frecuentemente citados en más de un 50\% de estudios. Las dimensiones de bienestar emocional, desarrollo personal y relaciones interpersonales aparecen referidas en un porcentaje menor, que oscila entre un $20 \mathrm{y}$ $30 \%$, aproximadamente. El resto de dimensiones, derecho, bienestar material y autodeterminación, sólo se encuentran en una décima parte de los estudios (Shalock y Verdugo, 2003).

Esta dificultad en su definición plantea otro tema candente, su evaluación. En estos últimos años, los desarrollos conceptuales y las implicaciones prácticas derivadas del propio constructo han impulsado el interés por los principios que han de guiar el proceso de evaluación (Verdugo y Schalock, 2006). El objetivo se centra en disponer de instrumentos con adecuados criterios de bondad que faciliten una adecuada medición de la CV. Existen actualmente variados instrumentos, entrevistas estructuradas, escalas, cuestionarios e inventarios, etc., cuyo ámbito de aplicación se centra fundamentalmente en personas con discapacidad jóvenes. La elaboración de estos instru- 
mentos ha tenido especial desarrollo en el ámbito de la discapacidad intelectual, siendo el Quality of Life Questionnaire de Schalock y Keith (1993) uno de los mejores instrumentos, ya adaptado a población española, en concreto para evaluar la calidad de vida percibida por adultos con discapacidad intelectual que desarrollan actividad laboral (Crespo, 2003). También contamos en nuestro entorno con la Escala Integral (Verdugo, Arias y Gómez, 2006), que permite la evaluación objetiva de calidad de vida en adultos jóvenes con discapacidad intelectual.

Sin embargo, la multidimensionalidad y subjetividad del concepto y la ausencia de una definición consensuada hacen que la evaluación de la CV se torne compleja (Cummins, 2005; Aguado, Alcedo, Rueda, González y Real, 2008). A esta dificultad se añade el hecho de que los intentos por medir este constructo no cuentan con una amplia y dilatada trayectoria, especialmente en la población de personas con discapacidad mayores, la población específica que nos ocupa. No contamos con instrumentos elaborados o adaptados para evaluar la CV de estas personas mayores con discapacidad que envejecen, población que presenta un progresivo incremento y que está planteando numerosos y novedosos retos. La relevancia social que va tomando este colectivo de personas mayores, en general, y de personas con discapacidad, en particular, especialmente en lo relativo a su trascendencia cuantitativa, es enorme. Más de la mitad de las personas con discapacidad que residen en nuestro país tiene 65 o más años. Entre 45 y 64 años unas 820.000 personas presentan discapacidades, lo que significa una cuarta parte del total de personas con discapacidad, personas que ya están haciendo frente a un proceso de envejecimiento prematuro. Ítem más, el "núcleo duro" de las situaciones de dependencia, es decir, el número de personas residen- tes en domicilios familiares con discapacidad severa y total para realizar actividades básicas de la vida diaria, es de 859.890 personas (IMSERSO, 2005). El riesgo estriba en no poder dar respuesta de calidad a sus necesidades ya que es mucho lo que desconocemos acerca de este colectivo. De hecho, es el grupo de edad de la población con discapacidad menos estudiado. La carencia de un marco conceptual que incluya tanto los aspectos propios del proceso de envejecimiento como los de la discapacidad, así como la ausencia de modelos teóricos sólidos y consensuados, no han posibilitado el desarrollo de lineas de investigación que aporten datos con buen respaldo empírico acerca de la CV de estas personas (Aguado, Alcedo, Fontanil, Arias, Verdugo y Badia, 2006).

Urge, pues, una clara delimitación conceptual del constructo de CV en la vejez. Dada su complejidad sabemos que es dificil hallar una definición unánimemente aceptada. Aunque existe cierto consenso en señalar su enfoque multidimensional que enfatiza el funcionamiento físico, la energía y vitalidad personal, el bienestar psicológico y emocional, el funcionamiento social, los apoyos recibidos y percibidos y la satisfacción con la vida, su evaluación se torna aún más compleja al no existir instrumentos suficientemente adecuados. Ante este panorama, es preciso optar por un determinado concepto y modelo de CV que nos permita elaborar y diseñar un instrumento de evaluación que cumpla con las directrices exigidas a nivel científico. A saber, i) tener como sustrato un sólido modelo teórico, ii) cumplir con las garantías de fiabilidad y validez demostradas y iii) aportar información útil para la puesta en marcha de medidas de apoyo y atención adecuadas que den respuesta a las necesidades de la población objeto de esta investigación, las personas con discapacidad que envejecen. 
En este sentido, la conceptualización de CV propuesta por Shalock, como un constructo multidimensional que abarca tanto aspectos objetivos como subjetivos, y que se refleja en una percepción general de bienestar o satisfacción, es la que consideramos como más adecuada para los objetivos que persigue nuestra investigación. De hecho, este modelo nos ha servido de base y guía en el diseño y elaboración de la Escala de Calidad de Vida, construida y aplicada dentro de una investigación más amplia ${ }^{1}$, y de la que seguidamente presentaremos sus propiedades psicométricas.

\section{METODOLOGÍA}

\section{Participantes}

La selección de los sujetos se hizo a través de un muestreo no probabilístico de carácter incidental o accidental. Se ha recurrido principalmente a las asociaciones y entidades públicas y privadas que están en contacto y atienden a los distintos colectivos de personas con discapacidad en cada una de las 16 comunidades autónomas donde se ha realizado el trabajo. Un equipo de colaboradores, principalmente licenciados en psicologia, estudiantes de segundo ciclo de dicha licenciatura y de educación especial, previamente entrenados para desarrollar el trabajo de campo, establecía el contacto con las instituciones y asociaciones y procedia a la aplicación de la escala.

Se ha establecido como punto de corte la edad de 45 años, nivel considerado como indicativo de envejecimiento en las personas con discapacidad. Aunque la edad cronológica es, en todo caso, una medida arbitraria y no muy precisa del proceso de envejecimiento, puesto que no hay un único momento en el que una persona se convierte en "mayor", hemos considerado adecuado tomar esta edad de 45 años como umbral del inicio de la vejez. Este punto de corte queda justificado por los siguientes motivos. En primer lugar, la frecuente y repetida idea de que el envejecimiento de algunas personas con discapacidad se adelanta a antes de los 50 años, traduciéndose fundamentalmente en un envejecimiento fisico prematuro, en ocasiones acompañado de disfunciones en lo psicológico y social, y reconocido por gran parte de los afectados y de los especialistas. En segundo lugar, nos hemos apoyado en la Encuesta sobre discapacidades, deficiencias y estado de salud: Año 1999 del INE (2000), que establece como uno de los niveles de análisis el rango de 45 a 64 años reconociendo este grupo de edad como el primer estadío de ese proceso de envejecimiento.

La escala ha sido cumplimentada por un total de 2.292 personas con discapacidad mayores de 45 años. La media de edad se sitúa en 59,26 años, con un rango que parte de los 45 y llega hasta los 99 años. En cuanto al género, la muestra es bastante homogénea, con porcentajes similares entre varones, $51,5 \%$, y mujeres, $48,5 \%$. Por lo que respecta a la residencia, la mayor parte de la muestra reside en zonas urbanas, $67,3 \%$. Atendiendo a la procedencia, se reparte principalmente entre las comunidades autónomas del Principado de Asturias, 45,8\%, Castilla-León, 17,4\%, y Euskadi, 12,2\%. En lo relativo al tipo de discapacidad (Tabla 1), existe un claro predominio de personas con discapacidad física, 37,3\%, y con discapacidad intelectual, 32,3\%. El resto de participantes se agrupa con una representa-

\footnotetext{
${ }^{1}$ Este trabajo forma parte de una investigación más amplia, que ha sido financiada por el IMSERSO, enmarcada dentro del ámbito recogido en la convocatoria del Plan Nacional I+D+I, bajo el acrónimo PREDEPENDIS (Referencia E-1/05).
} 
ción mucho menor en las demás categorías de discapacidad. Estas personas llevan con la discapacidad un tiempo medio de evolución de 37,03 años, con una desviación típica de 21,39 años. Asi, pues, contamos con sujetos que llevan un amplio periodo de edad conviviendo con su discapacidad, situación que les hace portadores de relevantes conocimientos acerca de la experiencia de vivir y envejecer desde esta perspectiva.

Por último, y en lo que atañe al tipo de convivencia (Tabla 2), observamos que más de la mitad de la muestra de nuestros discapacitados mayores, un 59,5\%, conviven en ambientes familiares, bien sea dentro de su núcleo familiar directo o el más próximo. En el ámbito residen- cial se encuentra un $28,5 \%$ de personas. Asi, pues, estamos en condiciones de afirmar que hemos conseguido restringir la representación de personas mayores residentes en ámbitos institucionales, ya que no representan a la población de personas con discapacidad mayores que, en nuestro país, y según datos recogidos en la encuesta del INE (Jiménez y Huete, 2002 , 2003), en su mayoría conviven dentro del ámbito familiar.

\section{Elaboración de la Escala de Calidad de Vida}

La versión inicial de la Escala de Calidad de Vida (ECV) consta de un total de 65 items o preguntas que muestrean las

Tabla 1. Distribución de la muestra por tipo de discapacidad

\begin{tabular}{|l|l|r|}
\hline \multicolumn{2}{|c|}{} & \\
\hline Válidos & Porcentaje \\
\cline { 2 - 2 } & Discapacidad & 32,3 \\
& intelectual & 37,3 \\
& Discapacidad física & 5,6 \\
& Deficiencia visual & 7,7 \\
& Deficiencia auditiva & 6,7 \\
& Trastornos mentales & 5,7 \\
& Alzheimer & 4,6 \\
& Parkinson & 100,0 \\
& Total &
\end{tabular}

Tabla 2. Distribución de la muestra por tipo de convivencia

\begin{tabular}{|l|l|r|}
\hline \multicolumn{2}{|l|}{} & \\
\hline Válidos & Sólo & 6,9 \\
& Familia propia & 35,3 \\
& Familia de origen & 24,2 \\
& Otras fórmulas & 5,0 \\
& Servicio residencial & 28,5 \\
& Total & 100,0 \\
\hline
\end{tabular}


distintas dimensiones de CV. El objetivo estriba en que la escala recoja la importancia y grado de utilización de los distintos indicadores objetivos de $\mathrm{CV}$ que definen las dimensiones del modelo propuesto por Schalock (1996). La versión definitiva de esta escala tras modificar, de acuerdo con la experiencia de las distintas fases de la investigación en las que se ha utilizado la escala, las respuestas dicotómicas en escalares para así obtener mayor precisión y variabilidad, consta de un número menor de items, 47 en total, dado que la verificación empírica de los datos ha aconsejado eliminar de los análisis aquellos items que no han alcanzado en esta muestra homogeneidad corregida suficiente. Es decir, se han eliminado 17 items que obtuvieron indices bajos 0 negativos de homogeneidad corregida. Por tanto, la ECV ha quedado conformada como una escala tipo Likert con 4 opciones de respuesta. Asimismo, y dada la relevancia que presentan determinadas características sociodemográficas y clínicas en el proceso de envejecimiento de estas personas, la escala recoge datos personales relacionados con dichas variables. Tras la elaboración de la escala y su correspondiente aplicación, el primer paso ha consistido en proceder a su validación para así conocer su aplicabilidad en personas

Tabla 3.1. Composición factorial de la Escala de Calidad de Vida

\begin{tabular}{|c|c|c|c|c|c|c|c|}
\hline FACTOR & PI & İTEM & M & DT & $\mathbf{r}$ & alfa & ALFA \\
\hline \multirow{4}{*}{ Factor 1} & 1 & Grado de autonomia en tareas domèsticas & 2,38 & 2,15 & 0,37 & 0,93 & \multirow{10}{*}{0,92} \\
\hline & 2 & Grado de autonomia en aclividades básicas & 3,15 & 2,15 & 0,48 & 0,92 & \\
\hline & 3 & Grado de autonomia para levanlar'se y acostarse & 3,34 & 2,16 & 0,39 & 0,93 & \\
\hline & 4 & Grado de autonomia para cambiar posición cuerpo & 3,55 & 2,11 & 0,40 & 0,93 & \\
\hline \multirow{6}{*}{$\begin{array}{l}\text { Bienestar } \\
\text { fisico }\end{array}$} & 5 & Grado de autonomia para moverse y desplazarse & 3,18 & 2,09 & 0,38 & 93 & \\
\hline & 6 & Grado de autonomia en el uso transporte puiblico & 2,40 & 2,37 & 0,31 & 0,93 & \\
\hline & 7 & Control de eslinteres & 3,11 & 2,85 & 0,29 & 0,93 & \\
\hline & 8 & Ejercicio lisico o aclividad deporlivá & 1,78 & 2,19 & 0,26 & 0,93 & \\
\hline & $\bar{g}$ & Calidad de hábitos alimenticios & 2,33 & 2,40 & 0,32 & 0,93 & \\
\hline & 10 & Valoractón personal de estado de salud & 2,68 & 2,28 & 0,34 & 0,93 & \\
\hline \multirow{8}{*}{$\begin{array}{c}\text { Factor } 2 \\
\text { IS } \\
\text { Inclusión } \\
\text { social }\end{array}$} & $\Pi 1$ & Práclica de hobby o aćlividad ocio & 2,15 & 2,15 & 0,35 & 0,93 & \multirow{8}{*}{0,69} \\
\hline & 12 & Formación de asociaciones de ayuda & 1,91 & 1,91 & 0,56 & 0,92 & \\
\hline & 13 & Información sobre recursos & 0,94 & 0,94 & 0,54 & 0,92 & \\
\hline & 14 & Intensidad o frecuencla de apoyos & 1,82 & 1,82 & 0,49 & 0,92 & \\
\hline & 15 & Participación en aclividades comunitarias & 1,71 & 1,71 & 0,20 & 0,93 & \\
\hline & 16 & Barreras arquiteciónicas de mi entorno & 3,32 & 3,32 & 0,15 & 0,93 & \\
\hline & 17 & Limitaciones de acceso a la vida comunitaria & 3,34 & 3,34 & 0,18 & 0,93 & \\
\hline & 18 & Sentimiento de respeto y aceptación por la sociedad & 2,38 & 2,28 & 0,34 & 0,93 & \\
\hline \multirow[b]{2}{*}{ Factor 3} & 19 & Persona como las demás & 2,28 & 3,06 & 0,67 & 0,92 & \multirow{8}{*}{0,91} \\
\hline & 20 & Importancia de la religión & 1,97 & 2,94 & 0,53 & 0,92 & \\
\hline \multirow{6}{*}{$\begin{array}{l}\text { BE } \\
\text { enestar } \\
\text { nocional }\end{array}$} & 21 & I'Tacticante & 1,75 & 2,92 & 0,48 & 0,92 & \\
\hline & 22 & Satisfaccion con la situaclon actual & 1,98 & 2,89 & 0,69 & 0,92 & \\
\hline & 23 & Problemas familiares importantes & 2,13 & 3,03 & 0,70 & 0,92 & \\
\hline & 24 & Salislacción con la vida al'eclivá & 2,16 & 2,97 & 0,72 & 0,92 & \\
\hline & 25 & Satisfacción con cl ambiente en que vive & 2,38 & 2,83 & 0,73 & 0,93 & \\
\hline & 26 & Satisfacción con la vida sexual actual & 0,01 & 4,62 & 0,42 & 0,93 & \\
\hline
\end{tabular}

Nota: "PI"; posición del item en la escala, "M"; media del ítem. "IT"; desviación típiça del item.

"r": cocliciente de comclación de cada ílcm con la escala total. "alfa": coclicicnte de fiabilidad de la escala si se climina cl ítcm. "ALFA": Fiabilidad del lactor. 
Escala de Calidad de Vida (ECV) para personas con discapacidad que envejecen: estudio preliminar

Tabla 3.2. Composición factorial de la Escala de Calidad de Vida (Continuación)

\begin{tabular}{|c|c|c|c|c|c|c|c|}
\hline FACTOR & PI & ÍTEM & M & DT & $\mathbf{r}$ & alfa & ALFA \\
\hline \multirow{4}{*}{$\begin{array}{c}\text { Factor } 4 \\
\text { DP }\end{array}$} & $\overline{27}$ & Capacidad para cuidado de si mismo & 2,68 & 0,93 & 0,37 & 0,93 & \multirow{8}{*}{0,85} \\
\hline & 28 & Capacidad para ocuparse bienestar familiar & 1,94 & 0,93 & 0,29 & 0,93 & \\
\hline & 29 & Capacidad para relacionarse y comunicarse & 3,04 & 0,93 & 0,40 & 0,93 & \\
\hline & 30 & $\begin{array}{l}\text { Toma de décisiones sobre actividades de la vida } \\
\text { diaria }\end{array}$ & 2,03 & 0,93 & 0,36 & 0,93 & \\
\hline \multirow{4}{*}{$\begin{array}{c}\text { Desarrollo } \\
\text { personal }\end{array}$} & 31 & Elecclön de dönde y con quilèn vivir & 1,73 & 0,93 & 0,34 & 0,93 & \\
\hline & 32 & Metas y objetivos en la vida & 1,30 & 0,92 & 0,63 & 0,92 & \\
\hline & 33 & Buen control personal & 1,82 & 0,92 & 0,74 & 0,92 & \\
\hline & 34 & Salislacción con la vida & 1,97 & 0,92 & 0,74 & 0,92 & \\
\hline \multirow{8}{*}{$\begin{array}{c}\text { Relaciones } \\
\text { interpersonales }\end{array}$} & 35 & Satisfacclón con las relaciones familiares & $\overline{2,23}$ & 2,99 & $\overline{0,43}$ & 0,93 & \multirow{8}{*}{0,88} \\
\hline & 36 & Salislacción con el apoyo lámiliar & 2,49 & 2,55 & 0,39 & 0,93 & \\
\hline & 37 & Salislacción con el láalo recibido & 2,12 & 3,43 & 0,54 & 0,92 & \\
\hline & 38 & Amigos & 2,49 & 2,28 & 0,50 & 0,92 & \\
\hline & 39 & Satisfacción con las relaciones de amistad & 2,17 & 2,98 & 0,39 & 0,92 & \\
\hline & 40 & Intimidad para relacionarse & 2,02 & 3,04 & 0,39 & 0,93 & \\
\hline & 41 & Relaciones sociales como los demás & 2,16 & 2,58 & 0,49 & 0,92 & \\
\hline & 42 & Salislacción con todas las rélaciones & 1,90 & 3,13 & 0,68 & 0,92 & \\
\hline \multirow[t]{5}{*}{ Factor 6} & 43 & Satisfacclón con ingresos actuales & 1,76 & 2,78 & 0,32 & 0,93 & \multirow{5}{*}{0,70} \\
\hline & 44 & Tenencia de vivienda propia & 1,75 & 2,90 & 0,28 & 0,93 & \\
\hline & 45 & Condiciones adecuadas de la vivienda & 1,74 & 3,81 & 0,54 & 0,92 & \\
\hline & 46 & Lugar de residencia adecuado & 2,30 & 2,75 & 0,26 & 0,93 & \\
\hline & 47 & Disposición de ayudas técnicas personales & 0,07 & 4,53 & 0,28 & 0,93 & \\
\hline
\end{tabular}

Nota: "Pl": posición del itcm cn la cscala. "M": media del itcm. "DI". desviación tipica del itcm.

"r": cocficiente de corrclación de cada itcm con la cscala total. "alfa": cocficiente de fiabilidad de la cscala si sc climina el ílcm. " $\Lambda L F \Lambda "$ "Fiabilidad del Factor.

con discapacidad que envejecen. Para ello y, en primer lugar, se ha realizado el análisis de la fiabilidad de la escala cuyos resultados presentamos en el siguiente apartado.

\section{Propiedades psicométricas de la Escala de Calidad de Vida}

\section{Datos sobre fiabilidad}

Se ha examinado la consistencia interna de la escala en la muestra de 2.292 personas con discapacidad mayores de 45 años utilizando el procedimiento de análisis específico inter-ítem (alfa de Cronbach). Todos los resultados aparecen de forma conjunta en las tablas 3.1 y 3.2 .
A su vez, en la tabla 4 se recoge por separado la correlación entre cada item y la escala total, el alfa que se obtendría si eliminamos cada uno de los ítems por separado y el alfa para el total de la escala.

La correlación entre cada ítem y la escala total arroja coeficientes próximos y superiores a 0,40, satisfaciendo así el criterio mínimo de 0,20 considerado como el más adecuado para este tipo de escalas. Estos resultados apuntan hacia una alta equivalencia funcional entre la respuesta a cada ítem y la respuesta al total de la escala. Así pues, y dado que los items presentan índices satisfactorios de correlación, se pone de manifiesto un adecuado grado de discriminación, lo que habla a favor de homogeneidad dentro de la escala de CV. 
Tabla 4. Índices de homogeneidad corregida de la ECV

\begin{tabular}{|c|c|c|c|}
\hline PI & İTEM & $\mathbf{r}$ & alfa \\
\hline 1 & Grado de autonomía en tareas domésticas & 0,37 & 0,93 \\
\hline 2 & Grado de autonomía en actividades básicas & 0,48 & 0,92 \\
\hline 3 & Grado de autonomía para levantarse y acostarse & 0,39 & 0,93 \\
\hline 4 & $\begin{array}{l}\text { Grado de autonomía para cambiar posición } \\
\text { cuerpo }\end{array}$ & 0,40 & 0,93 \\
\hline 5 & Grado de autonomia para moverse y desplazarse & 0,38 & 0,93 \\
\hline 6 & Grado de autonomía en el uso transporte público & 0,31 & 0,93 \\
\hline 7 & Control de esfinteres & 0,29 & 0,93 \\
\hline 8 & Ejercicio fisico o actividad deportiva & 0,26 & 0,93 \\
\hline 9 & Calidad de hábitos alimenticios & 0,32 & 0,93 \\
\hline 10 & Valoración personal de estado de salud & 0,34 & 0,93 \\
\hline 11 & Práctica de hobby o actividad ocio & 0,35 & 0,93 \\
\hline 12 & Formación de asociaciones de ayuda & 0,56 & 0,92 \\
\hline 13 & Información sobre recursos & 0,54 & 0,92 \\
\hline 14 & Intensidad o frecuencia de apoyos & 0,49 & 0,92 \\
\hline 15 & Participación en actividades comunitarias & 0,20 & 0,93 \\
\hline 16 & Barreras arquitectónicas de mi entorno & 0,15 & 0,93 \\
\hline 17 & Limitaciones de acceso a la vida comunitaria & 0,18 & 0,93 \\
\hline 18 & $\begin{array}{l}\text { Sentimiento de respeto y aceptación por la } \\
\text { sociedad }\end{array}$ & 0,34 & 0,93 \\
\hline 19 & Persona como las demás & 0,67 & 0,92 \\
\hline 20 & Importancia de la religión & 0,53 & 0,92 \\
\hline 21 & Practicante & 0,48 & 0,92 \\
\hline 22 & Satisfacción con la situación actual & 0,69 & 0,92 \\
\hline 23 & Problemas familiares importantes & 0,70 & 0,92 \\
\hline 24 & Satisfacción con la vida afectiva & 0,72 & 0,92 \\
\hline 25 & Satisfacción con el ambiente en que vive & 0,73 & 0,93 \\
\hline 26 & Satisfacción con la vida sexual actual & 0,42 & 0,93 \\
\hline 27 & Capacidad para cuidado de sí mismo & 0,37 & 0,93 \\
\hline 28 & Capacidad para ocuparse bienestar familiar & 0,29 & 0,93 \\
\hline 29 & Capacidad para relacionarse y comunicarse & 0,40 & 0,93 \\
\hline 30 & $\begin{array}{l}\text { Toma de decisiones sobre actividades de la vida } \\
\text { diaria }\end{array}$ & 0,36 & 0,93 \\
\hline 31 & Elección de dónde y con quién vivir & 0,34 & 0,93 \\
\hline 32 & Metas y objetivos en la vida & 0,63 & 0,92 \\
\hline 33 & Buen control personal & 0,74 & 0,92 \\
\hline 34 & Satisfacción con la vida & 0,74 & 0,92 \\
\hline 35 & Satisfacción con las relaciones familiares & 0,43 & 0,93 \\
\hline 36 & Satisfacción con el apoyo familiar & 0,39 & 0,93 \\
\hline 37 & Satisfacción con el trato recibido & 0,54 & 0,92 \\
\hline 38 & Amigos & 0,50 & 0,92 \\
\hline 39 & Satisfacción con las relaciones de amistad & 0,39 & 0,92 \\
\hline 40 & Intimidad para relacionarse & 0,39 & 0,93 \\
\hline 41 & Relaciones sociales como los demás & 0,49 & 0,92 \\
\hline 42 & Satisfacción con todas las relaciones & 0,68 & 0,92 \\
\hline 43 & Satisfacción con ingresos actuales & 0,32 & 0,93 \\
\hline 44 & Tenencia de vivienda propia & 0,28 & 0,93 \\
\hline 45 & Condiciones adecuadas de la vivienda & 0,54 & 0,92 \\
\hline 46 & Lugar de residencia adecuado & 0,26 & 0,93 \\
\hline 47 & Disposición de ayudas técnicas personales & 0,28 & 0,93 \\
\hline
\end{tabular}

Nota: "PI": posición del ítem en la escala; "r": coeficiente de correlación de cada item con la escala total. "alfa": coeficiente de fiabilidad de la escala si se elimina el item. 
Por lo que respecta a los índices alfa de consistencia interna, todos son elevados, con puntuaciones que oscilan entre 0,92 y 0,93 , obteniéndose un coeficiente global de 0,92 , lo que indicaría una alta homogeneidad. No debemos olvidar que a partir de 0,80 se considera que el coeficiente de consistencia es bueno, calificándose de excelente cuando supera el 0,90 .

En sintesis, la consistencia interna de la escala es alta, lo cual demuestra que es un instrumento fiable, es decir mide de forma adecuada la dimensión asignada como campo de exploración, la CV en relación a una serie de dimensiones básicas. El siguiente objetivo es el análisis dimensional de la ECV, es decir, su estructura factorial, aspecto que nos proporcionará datos acerca de su validez estructural.

\section{Estructura factorial}

Una vez analizada la fiabilidad de la ECV se procedió a su análisis factorial, otro de los objetivos planteados en esta investigación con el fin de conocer la aplicabilidad de la escala a la población de personas con discapacidad de edad. El procedimiento de factorización ha sido rotación promax sobre componentes principales.

En la tabla 5 aparecen las medias y las desviaciones típicas de los 47 items que forman la escala. Los resultados indican que existe variabilidad y dispersión de las puntuaciones y que las respuestas dadas a los items se adecuan a distribuciones normales. Los criterios seguidos para seleccionar los items que finalmente formarian parte de los factores fueron los siguientes:

- Se han considerado solo las saturaciones iguales o superiores a 0,35.
- Si un ítem satura significativamente en dos o más factores con el mismo signo, se tomará sólo en aquel factor en el que presente mayor saturación.

- Si un item presenta saturación en más de un factor con signo distinto se conserva el item en ambos factores.

- Cada uno de los factores debe estar formado al menos por tres items.

Siguiendo estos criterios se obtuvo una solución de seis factores, cuya composición detallamos a continuación.

El primer factor (tabla 6), formado por un total de 10 ítems, le hemos denominado "bienestar fisico", y muestrea indicadores relativos a la capacidad para la realización de actividades de la vida diaria, movilidad, salud y nutrición. Presenta una elevada consistencia interna, 0,92 .

El segundo factor (tabla 7), compuesto por 8 items, factor relativo a cuestiones que tienen que ver con la aceptación por parte de la sociedad, la participación en actividades comunitarias, el voluntariado y las redes sociales, principalmente, representa la dimensión de "inclusión social". El índice alfa de consistencia interna es aceptable, 0,69, aunque menor que el anterior factor. No obstante, también recoge menor número de ítems, lo que afecta al indice alfa que es una función del número de ítems.

El tercer factor (tabla 8), también formado por 8 items, presenta una alta homogeneidad, alcanzando su índice alfa el valor de 0,91. Sus ítems están referidos a indicadores de "bienestar emocional" tales como la satisfacción personal, felicidad, espiritualidad y afecto. 
Tabla 5. Medias y desviaciones típicas de los 47 items de la ECV ( $N=2.292)$

\begin{tabular}{|c|c|c|c|}
\hline PI & İTEM & $\mathbf{M}$ & DT \\
\hline 1 & Grado de autonomía en tareas domésticas & 2,38 & 2,15 \\
\hline 2 & Grado de autonomia en actividades básicas & 3,15 & 2,15 \\
\hline 3 & Grado de autonomia para levantarse y acostarse & 3,34 & 2,16 \\
\hline 4 & $\begin{array}{l}\text { Grado de autonomia para cambiar posición } \\
\text { cuerpo }\end{array}$ & 3,55 & 2,11 \\
\hline 5 & Grado de autonomía para moverse y desplazarse & 3,18 & 2,09 \\
\hline 6 & Grado de autonomia en el uso transporte público & 2,40 & 2,37 \\
\hline 7 & Control de esfinteres & 3,11 & 2,85 \\
\hline 8 & Ejercicio fisico o actividad deportiva & 1,78 & 2,19 \\
\hline 9 & Calidad de hábitos alimenticios & 2,33 & 2,40 \\
\hline 10 & Valoración personal de estado de salud & 2,68 & 2,28 \\
\hline 11 & Práctica de hobby o actividad ocio & 2,15 & 2,15 \\
\hline 12 & Formación de asociaciones de ayuda & 1,91 & 1,91 \\
\hline 13 & Información sobre recursos & 0,94 & 0,94 \\
\hline 14 & Intensidad o frecuencia de apoyos & 1,82 & 1,82 \\
\hline 15 & Participación en actividades comunitarias & 1,71 & 1,71 \\
\hline 16 & Barreras arquitectónicas de mi entorno & 3,32 & 3,32 \\
\hline 17 & Limitaciones de acceso a la vida comunitaria & 3,34 & 3,34 \\
\hline 18 & $\begin{array}{l}\text { Sentimiento de respeto y aceptación por la } \\
\text { sociedad }\end{array}$ & 2,38 & 2,28 \\
\hline 19 & Persona como las demás & 2,28 & 3,06 \\
\hline 20 & Importancia de la religión & 1,97 & 2,94 \\
\hline 21 & Practicante & 1,75 & 2,92 \\
\hline 22 & Satisfacción con la situación actual & 1,98 & 2,89 \\
\hline 23 & Problemas familiares importantes & 2,13 & 3,03 \\
\hline 24 & Satisfacción con la vida afectiva & 2,16 & 2,97 \\
\hline 25 & Satisfacción con el ambiente en que vive & 2,38 & 2,83 \\
\hline 26 & Satisfacción con la vida sexual actual & 0,01 & 4,62 \\
\hline 27 & Capacidad para cuidado de sí mismo & 2,68 & 0,93 \\
\hline 28 & Capacidad para ocuparse bienestar familiar & 1,94 & 0,93 \\
\hline 29 & Capacidad para relacionarse y comunicarse & 3,04 & 0,93 \\
\hline 30 & $\begin{array}{l}\text { Toma de decisiones sobre actividades de la vida } \\
\text { diaria }\end{array}$ & 2,03 & 0,93 \\
\hline 31 & Elección de dónde y con quién vivir & 1,73 & 0,93 \\
\hline 32 & Metas y objetivos en la vida & 1,30 & 0,92 \\
\hline 33 & Buen control personal & 1,82 & 0,92 \\
\hline 34 & Satisfacción con la vida & 1,97 & 0,92 \\
\hline 35 & Satisfacción con las relaciones familiares & 2,23 & 2,99 \\
\hline 36 & Satisfacción con el apoyo familiar & 2,49 & 2,55 \\
\hline 37 & Satisfacción con el trato recibido & 2,12 & 3,43 \\
\hline 38 & Amigos & 2,49 & 2,28 \\
\hline 39 & Satisfacción con las relaciones de amistad & 2,17 & 2,98 \\
\hline 40 & Intimidad para relacionarse & 2,02 & 3,04 \\
\hline 41 & Relaciones sociales como los demás & 2,16 & 2,58 \\
\hline 42 & Satisfacción con todas las relaciones & 1,90 & 3,13 \\
\hline 43 & Satisfacción con ingresos actuales & 1,76 & 2,78 \\
\hline 44 & Tenencia de vivienda propia & 1,75 & 2,90 \\
\hline 45 & Condiciones adecuadas de la vivienda & 1,74 & 3,81 \\
\hline 46 & Lugar de residencia adecuado & 2,30 & 2,75 \\
\hline 47 & Disposición de ayudas técnicas personales & 0,07 & 4,53 \\
\hline
\end{tabular}

Nota: "PI": posición del ítem en la escala. "M": media del ítem.

"DT": desviación típica del ítem. 
Tabla 6. Estructura factorial de la ECV con rotación promax $(\mathrm{N}=\mathbf{2 . 2 9 2})$. FACTOR 1

\begin{tabular}{|c|l|}
\hline PI & \multicolumn{1}{|c|}{ Factor 1: BF: Bienestar fisico } \\
\hline 1 & Grado de autonomía en tareas domésticas \\
\hline 2 & Grado de autonomia en actividades básicas \\
\hline 3 & Grado de autonomía para levantarse y acostarse \\
\hline 4 & Grado de autonomía para cambiar posición cuerpo \\
\hline 5 & Grado de autonomía para moverse y desplazarse \\
\hline 6 & Grado de autonomia en el uso transporte público \\
\hline 7 & Control de esfinteres \\
\hline 8 & Ejercicio físico o actividad deportiva \\
\hline 9 & Calidad de hábitos alimenticios \\
\hline 10 & Valoración personal de estado de salud \\
\hline
\end{tabular}

Tabla 7. Estructura factorial de la ECV con rotación promax ( $\mathrm{N}=\mathbf{2 . 2 9 2}$ ). FACTOR 2

\begin{tabular}{|l|l|}
\hline PI & \multicolumn{1}{|c|}{ Factor 2: IS: Inclusión social } \\
\hline 11 & Práctica de hobby o actividad ocio \\
\hline 12 & Formación de asociaciones de ayuda \\
\hline 13 & Información sobre recursos \\
\hline 14 & Intensidad o frecuencia de apoyos \\
\hline 15 & Participación en actividades comunitarias \\
\hline 16 & Barreras arquitectónicas de mi entorno \\
\hline 17 & Limitaciones de acceso a la vida comunitaria \\
\hline 18 & Sentimiento de respeto y aceptación por la sociedad \\
\hline
\end{tabular}

Tabla 8. Estructura factorial de la ECV con rotación promax (N = 2.292). FACTOR 3

\begin{tabular}{|c|l|}
\hline PI & \multicolumn{1}{|c|}{ Factor 3: BE: Bienestar emocional } \\
\hline 19 & Persona como las demás \\
\hline 20 & Importancia de la religión \\
\hline 21 & Practicante \\
\hline 22 & Satisfacción con la situación actual \\
\hline 23 & Problemas familiares importantes \\
\hline 24 & Satisfacción con la vida afectiva \\
\hline 25 & Satisfacción con el ambiente en que vive \\
\hline 26 & Satisfacción con la vida sexual actual \\
\hline
\end{tabular}

El cuarto factor (tabla 9), recoge asimismo 8 items, y le hemos denominado "desarrollo personal y autodeterminación". Refleja cuestiones relacionadas con la autonomia, control personal, autodirección y toma de decisiones. Presenta buena consistencia interna, alfa 0,85.
El quinto factor (tabla 10), en el que saturan un total de 8 items, agrupa indicadores relativos a la interacción con la familia, amistades, intimidad y apoyos. Es, por tanto, un factor que muestrea las "relaciones intepersonales”. Su indice de consistencia inter- 
Tabla 9. Estructura factorial de la ECV con rotación promax ( $N=2.292)$. FACTOR 4

\begin{tabular}{|c|l|}
\hline PI & \multicolumn{1}{|c|}{ Factor 4: DP: Desarrollo personal } \\
\hline 27 & Capacidad para cuidado de si mismo \\
\hline 28 & Capacidad para ocuparse bienestar familiar \\
\hline 29 & Capacidad para relacionarse y comunicarse \\
\hline 30 & $\begin{array}{l}\text { Toma de decisiones sobre actividades de la vida } \\
\text { diaria }\end{array}$ \\
\hline 31 & Elección de dónde y con quién vivir \\
\hline 32 & Metas y objetivos en la vida \\
\hline 33 & Buen control personal \\
\hline 34 & Satisfacción con la vida \\
\hline
\end{tabular}

Tabla 10. Estructura factorial de la ECV con rotación promax $(\mathrm{N}=2.292)$. FACTOR 5

\begin{tabular}{|c|l|}
\hline PI & \multicolumn{1}{|c|}{ Factor 5: RI: Relaciones interpersonales } \\
\hline 35 & Satisfacción con las relaciones familiares \\
\hline 36 & Satisfacción con el apoyo familiar \\
\hline 37 & Satisfacción con el trato recibido \\
\hline 38 & Amigos \\
\hline 39 & Satisfacción con las relaciones de amistad \\
\hline 40 & Intimidad para relacionarse \\
\hline 41 & Relaciones sociales como los demás \\
\hline 42 & Satisfacción con todas las relaciones \\
\hline
\end{tabular}

na refleja alta homogeneidad, alfa 0,88 .

El sexto factor (tabla 11), es el que agrupa un menor número de items, 5 en total, disminuyendo en consecuencia su indice de consistencia interna, alfa 0,70, indice moderado pero aceptable. El contenido de los ítems refleja la dimensión de "bienestar material" a través de cuestiones que informan sobre los ingresos económicos, posesiones y ayudas técnicas.

En suma, los seis factores obtenidos tras la realización del análisis factorial exploratorio formarian seis núcleos funcionales, referidos a necesidades fundamentales que representan las dimensiones de vida de la persona con discapacidad que envejece. En conformidad con el modelo de CV propuesto por Shalock,

Tabla 11. Estructura factorial de la ECV con rotación promax $(\mathrm{N}=2.292)$. FACTOR 6

\begin{tabular}{|c|l|}
\hline PI & \multicolumn{1}{|c|}{ Factor 6: BM: Bienestar material } \\
\hline 43 & Satisfacción con ingresos actuales \\
\hline 44 & Tenencia de vivienda propia \\
\hline 45 & Condiciones adecuadas de la vivienda \\
\hline 46 & Lugar de residencia adecuado \\
\hline 47 & Disposición de ayudas técnicas personales \\
\hline
\end{tabular}


modelo que nos ha servido de guía para el diseño y elaboración del instrumento de evaluación que estamos validando, la Escala de Calidad de Vida que hemos factorializado, aglutina factores relacionados con el bienestar físico, inclusión social, bienestar emocional, desarrollo personal y autodeterminación, relaciones interpersonales y bienestar material. Estos indicadores, considerados como determinantes para llevar a cabo una adecuada evaluación de la $\mathrm{CV}$, permiten hacer más operativa la definición del constructo y concretan más su medición.

\section{CONCLUSIONES}

La CV y las necesidades percibidas son conceptos que han cobrado gran importancia a lo largo de estas últimas décadas en el ámbito de la discapacidad. Sin embargo, aún desconocemos mucho acerca de estos temas en la etapa de la vejez. Existe un predominio de publicaciones teóricas y la investigación empírica casi se encuentra a un nivel precientífico. La necesidad de disponer de instrumentos de evaluación de CV para este colectivo de personas con discapacidad mayores, que nos permitan realizar medidas fiables y válidas sobre las que planificar actuaciones y estrategias de intervención eficaces, y supervisar practicas sociales y apoyos para mejorar los resultados, ha orientado la elaboración y validación de la Escala de Calidad de Vida que acabamos de presentar.

El primer objetivo ha sido comprobar la fiabilidad de la escala. Para la realización de los análisis de fiabilidad se ha calculado el coeficiente de consistencia interna a través del alfa de Cronbach. Los resultados encontrados, tanto en el caso de los índices de homogeneidad corregida (alfa obtenido eliminando cada una de las variables), como en la escala global, muestran coeficientes elevados (puntuaciones alfa entre 0,92 y 0,93) que ponen de manifiesto que la escala cumple con los criterios de fiabilidad necesarios.

Una vez comprobada la fiabilidad se procedió al análisis factorial de la escala. A través del método de componentes principales con rotación promax se obtuvo una solución hexafactorial que muestrea seis de las principales dimensiones de CV propuestas por Schalock. En concreto, bienestar físico, inclusión social, bienestar emocional, desarrollo personal $\mathrm{y}$ autodeterminación, relaciones interpersonales y bienestar material. Estos resultados apoyan la validez de constructo de la escala y evidencian la adecuación de la misma a la hora de evaluar lo que tiene que medir, la CV tal y como es percibida por la muestra de nuestro estudio, las personas con discapacidad que envejecen.

En posteriores trabajos es preciso proceder a la realización de estudios centrados en el análisis de la validez concurrente de la ECV, así como análisis diferenciales en función de variables predictoras tales como tipo de discapacidad, grado de discapacidad, edad, género, tipo de convivencia, etc., para comprobar si estas variables clínicas y sociodemográficas que presentan gran relevancia en el proceso de envejecimiento de estas personas, establecen diferencias importantes en las distintas dimensiones de $\mathrm{CV}$ muestreadas por la escala.

En sintesis, la ECV recoge una serie de principios básicos para la medición de este aspecto determinante de una vida de calidad (Verdugo y Schalock, 2006). A saber, sus items muestrean aspectos relacionados con experiencias vitales de la persona con discapacidad que envejece, incluye dimensiones que dan carácter integral al sujeto, ya sean objetivos y subjetivos, valora el grado en que estas distin- 
tas dimensiones contribuyen a una vida plena y satisfactoria, y tiene en cuenta los distintos contextos en los que se desenvuelve la persona. Podemos por tanto abo- gar por la idoneidad de la ECV como instrumento de evaluación de la CV de las personas con discapacidad mayores desde una perspectiva multidimensional.

\section{BIBLIOGRAFÍA}

Aguado, A.L., Alcedo, M.A., Fontanil, Y., Arias, B., Verdugo, M.A. y Badia, M. (2006). Prevención de la dependencia y promoción de la autonomía personal: Estudio sobre el incremento de necesidades $y$ el descenso de calidad de vida en el proceso de envejecimiento prematuro de las personas con discapacidad. Informe de proyecto de investigación IMSERSO y Departamento de Psicologia, Universidad de Oviedo. (Inédito).

Aguado, A.L., Alcedo, M.A., Rueda, M.B., González, M. y Real, S. (En prensa). La evaluación e intervención de mayores dependientes. En R. Fernández-Ballesteros (Dir): Psicogerontología aplicada. Madrid: Sintesis.

Aguado, A.L., González, I., Alcedo, M.A. y Arias, B. (2003). Calidad de vida y lesión medular. En M.A. Verdugo y B. Jordán de Urries (Coors): Investigación, innovación y cambio, (ps. 623-664). Salamanca: Amarú Ediciones.

Crespo, M. (2003). Validación y aplicación de la Escala de Calidad de Vida de Schalock y Keith (1993) a adultos con discapacidad intelectual en contextos laborales. Tesis Doctoral. Departamento de Personalidad, Evaluación y Tratamiento Psicológicos. Universidad de Salamanca.

Cummins, R.A. (2005). Instruments assessing quality of life: characteristics and functions. En J.H. Hogg y A. Langa (Eds): Approaches to the assessment of adults with intellectual disabilities. Part. I. Londres: Blackwell.

IMSERSO, (2005). Libro Blanco de la Dependencia, Tomos I y II. Madrid: Ministerio de Trabajo y Asuntos Sociales.

INE (2000). Encuesta sobre discapacidades, deficiencias y estado de salud: Año 1999. Avance de resultados. Madrid: Instituto Nacional de Estadística.

Jiménez, A. y Huete, A. (2002). La discapacidad en cifras. Madrid: IMSERSO.

Jiménez, A. y Huete, A. (2003). Las discapacidades en España. Datos estadísticos. Aproximación desde la Encuesta sobre discapacidades, deficien- cias y estado de salud de 1999. Madrid: Real Patronato sobre Discapacidad.

Keith, K.D. (2001). International quality of life: Current conceptual, measurement and implementation issues. International Review of Research in Mental Retardation, 24, 49-74.

Schalock, R. L. (1996). Quality of Life. Vol. 1: Its Conceptualization, Measurement and Use. Washington, D.C.: American Association on Mental Retardation.

Schalock, R.L. (2005). Introduction and overview to the special issue. Journal of Intelectual Disability Research, 49 (9), 695-698.

Schalock, R.L. y Keith, K.D. (1993). Quality of Life Questionnaire Manual. Worthington, Ohaio: IDS Publishing Corporation.

Schalock, R.L. y Verdugo, M.A. (2003). Calidad de vida: Manual para profesionales de la educación, salud y servicios sociales. Madrid: Alianza Editorial. (Versión castellana de Handbook on Quality of Life for Human Service Practitioners. Washington, DC: American Association on Mental Retardation, 2002).

Schalock, R.L. y Verdugo, M.A. (2006). Revisión actualizada del concepto de calidad de vida. En M.A. Verdugo (Dir): Cómo mejorar la calidad de vida de las personas con discapacidad. Instrumentos y estrategias de evaluación, (ps.29-41). Salamanca: Amarú.

Verdugo, M. A., Arias, B. y Gómez, L. E. (2006). Escala integral de medición subjetiva y objetiva de la calidad de vida en personas con discapacidad intelectual. En M.A. Verdugo (Dir.): Cómo mejorar la calidad de vida de las personas con discapacidad. Instrumentos y estrategias de evaluación, (ps. 417-448). Salamanca: Amarú.

Verdugo, M.A. y Schalock, R.L. (2006). Aspectos clave para medir la calidad de vida. En M.A. Verdugo (Dir): Cómo mejorar la calidad de vida de las personas con discapacidad. Instrumentos y estrategias de evaluación, (ps.43-58). Salamanca: Amarú. 